\title{
Molecular mechanisms of drug-induced hearing loss
}

\author{
Jochen Schacht \\ Kresge Hearing Research Institute, University of Michigan, Ann Arbor, MI 48109, U.S.A.
}

\begin{abstract}
Although the ototoxic actions of a variety of drugs have long been documented, the biochemical mechanisms underlying such toxicity largely remain to be established. For example, recent advances have provided us with information about the actions of salicylates (aspirin) and diuretics (furosemide) but we are not yet able to specify the mechanisms by which these drugs damage the cochlea. On the other hand, the considerable amount of biochemical and pharmacological data on the effects of aminoglycosides (streptomycin, neomycin, gentamicin and related compounds) has enabled us to formulate a rational hypothesis of their mechanism of action.

We have previously presented evidence for an involvement of polyphosphoinositides in the ototoxic actions of aminoglycosides. Recent electrophysiological and pharmacokinctic studies have shown in addition that aminoglycosides occupy at least two distinct compartments in the course of their actions. Further studies of drug uptake in vitro and of drug toxicity in cochlear perfusions suggested the involvement of an active (energy-requiring) aminoglycoside transport system. These and other data are compatible with the following multi-step model of aminoglycoside toxicity:

(1) The initial step in the reaction sequence is an electrostatic interaction of aminogiycosides with the plasma membrane. The resulting displacement of calcium accounts for acute effects but the action is reversible and antagonized by divalent cations.

(2) An energy-dependent uptake process is required for the expression of toxicity. It can be prevented by select metabolic blockers.

(3) A crucial step in subsequent intracellular drug actions is the binding of aminoglycosides to phosphatidylinositol bisphosphate inhibiting its hydrolysis and preventing its physiological function. Hydrolysis of phosphatidylinositol bisphosphate is believed to be the key reaction in a fundamental transducing mechanism initiated by a number of physiological stimuli and resulting in a signal cascade to mobilize intracellular calcium stores.

(4) Other reactions possibly regulated by polyphosphoinositides (such as the synthesis of prostaglandins and the polymerization of actin) are potentially affected by aminoglycoside action.

(5) Aminoglycosides may interfere with further intracellular reactions which are controlled by calcium or polyamines.
\end{abstract}

\section{Introduction}

The awareness that certain drugs in clinical use may cause temporary or permanent disorders of the auditory or vestibular systems goes back at least a century. At that time the toxic effects of quinine, the salicylates and oil of chenopodium had been described in the scientific literature (North, 1880; Schwabach, 1884). Since then the number of drugs potentially interfering with the function of the labyrinth has grown considerably. One of the intriguing aspects of this phenomenon is that ototoxic drugs fall into several unrelated therapeutic classes. In addition to the analgesics and antipyretics (salicylates, quinine) there are the antibacterial aminoglycosides (for example, neomycin, gentamicin) and polypeptide antibiotics (viamycin, vancomycin), some metallo compounds (arsenicals, mercurials), 'loop' diuretics (ethacrynic acid, furosemide) and antineoplastic drugs (cis-dichlorodiammin-platinum). There is no apparent structural relationship between these drugs nor is there a predictable pattern of the pathology they may induce. Some act preferentially on the cochlea. some on the vestibular system, and within the cochlea they may act on the organ of Corti or the stria vascularis. The types of cellular damage they induce are also quite dissimilar as are the manifestations of toxicity such as tinnitus, temporary or permanent hearing loss (for a review of the pathology of drug-induced ototoxicity see Hawkins. 1976, and Wersäll, 1986).

Considering these multifaceted drug actions, it is not surprising that a unifying hypothesis of ototoxicity does not exist. Each drug has to be considered separately and generalizations cannot 
be made. Furthermore, to date only few of these drugs have been investigated for the molecular mechanism of their toxicity, among them most notably the aminoglycoside antibiotics. In addition, some recent information on the pharmacology and biochemistry of diuretic- and salicylateinduced ototoxicity (Rybak et al., 1984; Escoubet et al., 1985) shows promise of leading to a rational understanding of their action.

The aminoglycosides, still widely used for their antibacterial efficacy, were discovered in the 1940s (see Waksman, 1953) and their toxicity was described shortly after the first clinical application for the treatment of tuberculosis. Drugs of this class induce both nephrotoxicity which is generally reversible, and ototoxicity which is generally irreversible. Some of the aminoglycosides preferentially damage the cochlea (for example, amikacin), some the vestibular apparatus (streptomycin), while others show only gradual, if any predilections (gentamicin, tobramycin). The ototoxicity of the aminoglycosides will be the focus of our discussion in this chapter.

\section{Results and Discussion}

\section{Pharmacokinetics}

The first systematic investigations into aminoglycoside ototoxicity were pharmacokinetic studies of the drug distribution in the perilymph of animals (Voldrich, 1965; Vrabec et al., 1965; Stupp, 1970). This approach - analysis of an extracellular fluid rather than the affected tissues - is only indircet and its justification may have been the technical feasibility at the time. It had, however, considerable impact as these first studies seemed to show an 'accumulation' of the aminoglycosides in perilymph which was postulated to be the primary cause of their toxicity and organ specificity.

Recent detailed and well-controlled pharmacokinetic investigations, however, demonstrated that an accumulation of gentamicin in the inner ear fluids over plasma levels did not occur (Tran Ba Huy et al.. 1981, 1983, 1985). Furthermore, the concentrations of several aminoglycosides attained in inner ear fluids were shown to be unrelated to the resultant toxic effects (Ohtsuki et al., 1982; Ohtani et al., 1985). Conversely, direct application of different aminoglycosides in identical concentrations into the cochlea by perilymphatic per- fusion produced a differential loss of the cochlear microphonic potential, indicating that toxicity was largely an intrinsic property of the drugs rather than related to their concentration in inner ear fluids (Lodhi et al., 1980; Anniko et al., 1982). In addition to studies of inner ear fluids the determination of neomycin levels (Desrochers and Schacht, 1982) and gentamicin pharmacokinetics (Tran Ba Huy et al., 1986) in inner ear tissues showed that the concentration the drug reached in these tissues was not higher than in organs that do not show pathologies such as liver or lung.

The combined evidence, then, seems to rule out a mechanism of toxicity and organ specificity based solely or primarily on drug concentrations reached in inner ear fluids or lissues. This does not preclude the existence of drug uptake or transport systems in the affected cells. It means, however, that the mechanism (or mechanisms) of toxicity must lie in the specific biochemical interactions of the drugs with their target cells.

\section{Biochemical studies}

A major problem that arises in the study of any drug-induced pathology is the need to differentiate between primary and secondary actions. For example, after the initial insult to a cell by a drug a wide variety of biochemical pathologies may be observed that result not from a direct interaction with the drug itself but from the metabolic response of the cell to the primary insult. Such pathologies may be part of the chain of events leading to cell death or, in contrast, may be cellular repair mechanisms. In either case, they do not reflect the specific mechanism of drug action. It is the specific site(s) that need to be elucidated for a rational hypothesis of drug action.

Such a rational hypothesis of aminoglycoside action has been lacking until now. Not because of a lack of demonstrable biochemical effects but paradoxically an abundance of them. Aminoglycosides have been reported to affect DNA, RNA and protein synthesis, enzymes of energy metabolism and transport, gangliosides, mucopolysaccharides and lipids - virtually all categories of cellular components. Yet these findings have not led to a coherent explanation of the toxic mechanism probably hecause most of them are linked to secondary effects. 


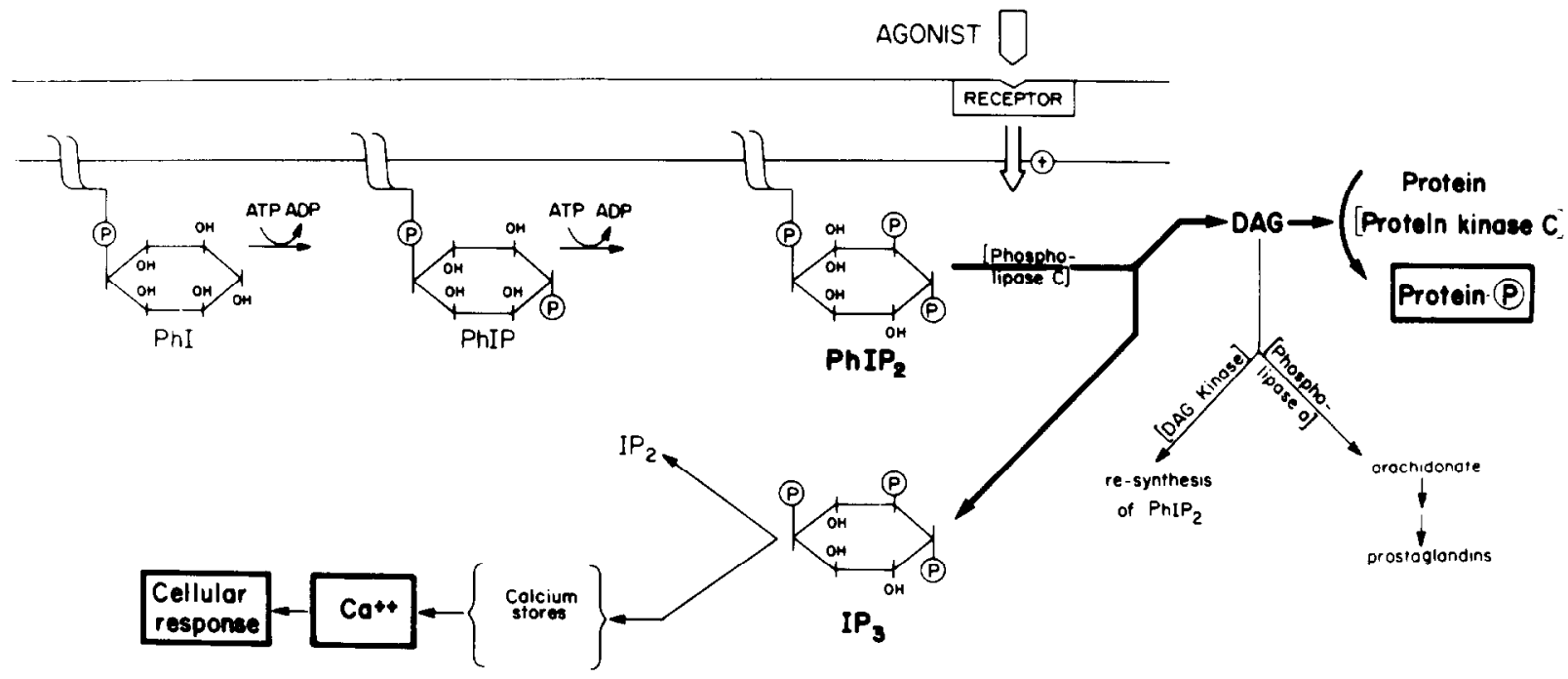

Fig. 1. The phosphoinositide cascade. Binding of the agonist to its receptor at the outer plasma membrane stimulates the phosphodiesteratic cleavage of phosphatidylinositol bisphosphate $\left(\mathrm{PhIP}_{2}\right)$ located in the inner plasma membrane. The resulting products, diglyceride (diacylglycerol, DAG) and inositol trisphosphate $\left(\mathrm{IP}_{3}\right)$ act as second messengers and elicit the physiological response of the cell via activation respectively, of a protein kinase and the release of $\mathrm{Ca}^{2+}$ from intracellular stores. The action of $\mathrm{IP}_{3}$ is terminated by its hydrolysis to inositol bisphosphate $\left(\mathrm{IP}_{2}\right)$, and $\mathrm{PhIP}_{2}$ is resynthesized from DAG via phosphatidic acid, CDP diglyceride, phosphatidylinositol ( $\mathrm{PhI}$ ) and phosphatidylinositol phosphate (PhIP). Synthesis of prostaglandins may also proceed from DAG. After Nishizuka (1984) and Berridge (1984).

We have previously presented evidence for an involvement of polyphosphoinositides in the toxic actions of aminoglycosides. Polyphosphoinositides are acidic phospholipids of the plasma membrane synthesized in two sequential phosphorylation reactions from phosphatidylinositol (Fig. 1). Phospholipids provide the structural framework of cell membranes, regulate membrane function and mediate the communication of the cell with the external environment.

All environmental stimuli are encoded at the cell membrane into molecular signals that traverse the membrane and elicit the appropriate physiologic response in the cell. Recent evidence suggests that polyphosphoinositides, which are located on the cytoplasmic site of the plasma membrane, constitute a fundamental transmembrane signaling system for neuromodulators and hormones which use calcium as an ultimate cellular messenger (Nishizuka, 1984; Berridge, 1984; Fig. 1). The initial step in this action is a stimulation of the phosphodiesteratic hydrolysis of membrane-bound phosphatidylinositol bisphosphate liberating diacylglycerol and inositol trisphosphate as second messengers and initiating an intracellular signal cascade. In this cascade, diacylglycerol activates a specific protein kinase $\mathrm{C}$, and inositol trisphosphate mobilizes intracellular calcium, two signals that trigger the cellular response. Sensory stimuli, e.g. visual excitation, may be processed by this mechanism (Brown et al., 1984).

Whether cochlear phosphoinositide metabolism is responsive in such a fashion remains to be established. We have investigated phospholipids in the mature and developing mammalian cochlea, in the otocyst in organ culture, in the fish and the frog lateral line and in the ear of the Noctuid moth (Stockhorst and Schacht, 1977; Kilian and Schacht, 1980; Anniko and Schacht, 1981; Tachibana et al., 1983; Schacht, 1984). All hair-cell systems contain polyphosphoinositides and the incorporation of radioactive $\left[{ }^{32} \mathrm{P}\right]$ orthophosphate consistently shows a fast kinetics and isotopic equilibrium indicative of the high rate of turnover of these lipids. Sound induced an increase in ${ }^{32} \mathrm{P}$ labeling of phosphatidylinositol phosphate and phosphatidylinositol bisphosphate in the ear of the Noctuid moth (Kilian and Schacht, 1980). Furthermore, the 
polyphosphoinositide cascade can be speculated to be involved in several aspects of auditory transduction, based on modulation of membrane properties and structural elements.

Extensive in vivo and in vitro studies from our laboratory have provided evidence that phosphatidylinositol bisphosphate may be a specific binding site for aminoglycosides (Schacht et al., 1977; Schacht, 1979). The configuration of the negative charges of phosphatidylinositol bisphosphate allows a specific three-point binding to the positively charged aminoglycoside. Hydrogen bonding may further stabilize the complex and other cationic groups of the antibiotic may 'anchor' the complex in the cell membrane at secondary sites. Due to this binding the metabolism of phosphatidylinositol bisphosphate is inhibited by aminoglycosides both in vivo (Orsulakova et al., 1978; Stockhorst and Schacht, 1977) and in vitro (Lodhi et al., 1979). In addition, specific changes in membrane structure and fluidity were induced by neomycin in lipid monolayers and liposomes which contained phosphatidylinositol bisphosphate (Lodhi et al., 1979; Wang et al., 1984a.b).

Following our initial reports on altered phosphoinositide metabolism in the neomycin-intoxicated ear and kidney (Schacht, 1974; Schibeci and Schacht. 1977) confirmation of aminoglycoside/ phosphoinositide interactions has come from a number of laboratories studying cochlear and renal lipid metabolism and physicochemical models. Incorporation of ${ }^{32} \mathrm{P}$ into cochlear polyphosphoinositides was enhanced by sound and depressed by neomycin (Yanagisawa et al., 1982). Aminoglycoside-induced altered renal phospholipid metabolism, particularly that of phosphoinositides, was reported (Koloyanides and PastorizaMuñoz, 1980; Marche et al., 1983). A specific interaction between neomycin and the 5'-phosphomonoester of phosphatidylinositol bisphosphate was determined by ${ }^{31} \mathrm{P}$ nuclear magnetic resonance (Hayashi et al., 1980). Finally, a series of aminoglycosides suppressed $\mathrm{K}^{+}$permeation in bilayer membranes formed by lyso-phosphatidylinositol bisphosphate and the degree of suppression correlated with the toxicity of the drugs (Sokabe et al.. 1982).

\section{Additional observations}

While the involvement of phosphatidylinositol bisphosphate in the toxicity of aminoglycosides thus seems well established a number of additional observations remained to be incorporated into a molecular model of ototoxicity.

First, recent electrophysiological and pharmacokinetic studies from our laboratory demonstrated that aminoglycosides occupy at least two distinct cellular compartments in the course of their toxic action. When gentamicin was applied to the cochlea by perilymphatic perfusion, the loss of the cochlear microphonic potential could be prevented by the simultaneous or immediately following addition of calcium (Takada and Schacht, 1983). This was in good agreement with an aminoglycoside/calcium antagonism which we had demonstrated in natural and artificial membranes (Lodhi et al., 1976). However, if the application of calcium was delayed, the decrease of the cochlear microphonics could no longer be reversed. This differentiated an acute and reversible phase of aminoglycoside toxicity from a non-reversible one. A similar observation of a biphasic action of streptomycin had been made by Wersäll and Flock (1964) in the lateral line organ of the fish.

Pharmacokinetic studies of gentamicin uptake into and release from inner ear tissues (Tran Ba Huy et al., 1986) provided independent evidence for the occupation of at least two cellular compartments by the drug. After both short-time infusion and chronic injections, gentamicin reached similar steady-state levels in the cochlea. The short-time treatment was not associated with signs of ototoxicity and the drug was released from the tissues with a half-life of 8 days. After chronic injections ototoxicity developed and the drug persisted in the tissues with a half-life of 36 days. Clearly, this difference in half-lives indicated the release of the drug from two different binding sites or compartments, although these need not be identical to those observed in the perfusion study.

If several compartments for drug binding exist the question arises as to their location and the process by which they are linked. One possible constellation is an initial drug action on the plasma membrane (representing the acute and reversible phase) followed by entry of the drug into the cell and intracellular actions (representing the chronic 
phase). Presently little is known about the mechanisms of entry into cochlear cclls of aminoglycoside antibiotics. This is quite in contrast to the well-researched uptake of these drugs into bacterial cells (Hancock, 1982) where energy-dependent processes have been established and into kidney where transport processes have been somewhat characterized (Josepovitz et al., 1982; Bennett et al., 1982).

The hypothesis that an energy-dependent uptake process may be a necessary step in aminoglycoside action comes from studies of gentamicin toxicity in cochlear perfusions and mechanisms of its prevention (Takada et al., 1985). If a bolus of gentamicin is administered during a time when the endolymphatic potential is depressed by anoxia or drugs the expected loss of the cochlear microphonic potential is prevented. In the most general interpretation, this signifies that a process dependent on normal energy metabolism is required for the expression of toxicity such as an uptake driven by an active transport or sym-port mechanism or by the transmembrane potential. An energy-dependent uptake of aminoglycosides is also, at least partially, operating in the crista ampullaris. A block of metabolism by low temperature or drugs prevented a significant portion of gentamicin uptake in vitro (Williams and Schacht, in preparation).

\section{The hypothesis (Fig. 2)}

The data elaborated by our laboratory are consistent with the following multi-step model of aminoglycoside toxicity.

(1) The first step in the reaction sequence is an electrostatic interaction of the aminoglycoside with negatively charged components of the outer plasma membrane. The resulting displacement of calcium accounts for the acute effects of the drug and the action is reversible and antagonized by cations.

(2) The drug is taken up into the cell by an energy-dependent transport process. This step can be prevented by metabolic blockers.

(3) The next and crucial step in the subsequent intracellular actions is the binding of the drug to phosphatidylinositol bisphosphate. The formation of the drug-lipid complex has two consequences: first, it prevents the hydrolysis of phosphatidylinositol bisphosphate which is the key reaction

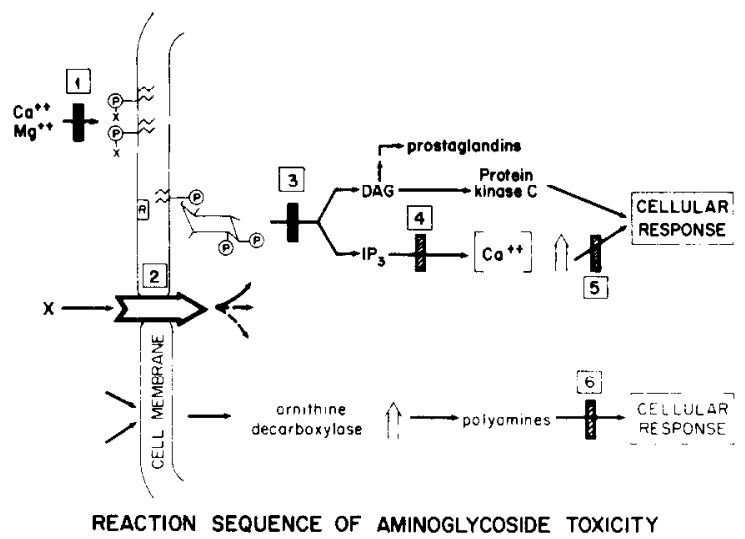

Fig. 2. Hypothesis of aminoglycoside toxicity. (1) Aminoglycosides bind to negatively charged sites on the plasma membrane. (2) They are taken up into the cell by an active transport system. (3) They form a complex with phosphatidylinositol bisphosphate and inhibit its hydrolysis. (4-5) They interfere with further steps of the phosphoinositide cascade and (6) other intracellular reactions. Solid bars and lines: documented aminoglycoside actions; hatched: postulated actions. Abbreviations as in Fig. 1. For details, see text.

in the phosphoinositide second messenger signal cascade; secondly, it disturbs membrane integrity and structure resulting in non-specific permeabilities of the membrane to ions and possibly to the antibiotic itself, causing an 'autocatalytic' entry of the drug into the cell.

(4) This binding would also inhibit other reactions regulated by polyphosphoinositides such as the polymerization of actin (Burn et al., 1985; I assing and I indberg, 1985) and the synthesis of prostaglandins. The inhibition of the latter has been documented recently (Escoubet et al., 1985).

(5) Once inside the cell, the aminoglycosides may interfere with further intracellular reactions as amply documented in the literature. This interference may be based on competition with divalent cations or polyamines or on binding to negatively charged compounds.

\section{Critical considerations}

To the best of our knowledge the hypothesis presented is compatible with data presented not only by our but also other laboratories to date. Nevertheless, several questions remain to be discussed. 
The first question is why, in the face of such a multistep reaction, the binding to polyphosphoinositides is considered a crucial step. The most convincing answer comes from the study of physicochemical models of drug-lipid interactions. In monomolecular films containing phosphatidylinositol bisphosphate the disturbance of membrane structure (measured as an increase in surface pressure) shows an excellent correlation with the ototoxicity of various aminoglycosides (Wang et al., 1984b; Fig. 3). Such a correlation, in particular the correct ranking of netilmicin and neamine, is not achieved with models that are based on lipids other than phosphatidylinositol bisphosphate strongly suggesting a direct relationship between polyphosphoinositide binding and toxicity.

A second question is the tissue specificity of the drug action. Neural tissue (including the ear) shows an active metabolism of phosphoinositides as does the kidney, while other tissues (such as liver or lung) seem less active. In both the inner ear and the kidney polyphosphoinositides may thus be a crucial determinant of toxicity. However, for the drug to exert this and other intracellular actions an entry into the cell is required. The susceptibility of an organ to aminoglycosides then may be de-

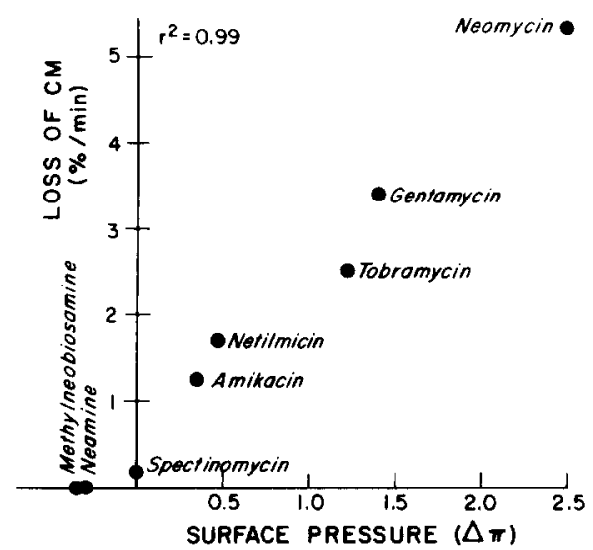

Fig. 3. Correlation of aminoglycoside ototoxicity with action on phosphatidylinositol bisphosphate. Aminoglycoside antibiotics and derivatives were tested for ototoxicity by cochlear perfusions (plotted on the ordinate) and for their interaction with a monomolecular film containing phosphatidylinositol bisphosphate (plotted on the abscissa). For details, see text and Wang et al. (1984b). termined by a combination of at least two factors: the presence of an active uptake system and the presence of a physiologically important population of phosphoinositides.

Another question and one of utmost clinical importance is the prevention of aminoglycoside-induced hearing loss. Pharmacologic treatments for detoxification or amelioration of ototoxicity have long been tried but have largely been unconvincing. It seems that an approach to prevention or amelioration of toxicity based on the hypothesis of membrane interactions may be more promising. Reversibility of initial aminoglycoside damage is a postulate of our hypothesis and supported by experimental data (Wersäll and Flock, 1964; Shiozawa and Yanagisawa, 1979; Kroese and van den Bercken, 1982) but less so by clinical experience (Matz, 1975). It should be noted, however, that cases of recovery of patients from gentamicininduced hearing loss have been reported (Jackson and Arcieri, 1971; Federspil, 1971; Tjernström et al., 1973) as well as from streptomycin and kanamycin damage, particularly when drug treatment was stopped shortly after the onset of symptoms (Federspil, 1979). The paucity of support for reversibility after parenteral treatment with aminoglycosides may be explained by our in-situ studies showing the reversibility limited to a narrow set of conditions determined by both duration and concentration of drug treatment ('lakada et al., 1983). Under appropriate conditions, calcium should be an effective antagonist in the early phase of toxicity. Indeed, Humes et al. (1984) reported that a dietary calcium load lowered gentamicin nephrotoxicity: excess serum calcium is eliminated by the kidney and competes with renal binding of aminoglycosides. It is interesting that calcium levels in the perilymph can be elevated by intravenous $\mathrm{CaCl}_{2}$ injections (Juhn and Youngs, 1976). Whether such transiently increased levels of cochlear calcium might affect aminoglycoside binding and ototoxicity remains to be established

\section{Conclusion}

Some unanswered, yel important questions remain. Why is there no strict correlation between nephro- and ototoxicity? Why do some of the drugs cause more damage to the cochlea than to 
the vestibular system and vice versa? Why are outer hair cells more susceptible than inner hair cells? These questions do in no way invalidate our model: they simply point to the fact that drug reactions are usually complex and models necessarily simple. Speculation about answers can first point to the combined requirement of an uptake system and phosphoinositides for the maximal expression of toxicity. If one or the other or both are absent from a cell, entirely different patterns of toxicity can be expected. In addition, calcium- or polyamine-regulated intracellular processes may be an important part of the overall mechanism and these still need to be investigated. In this context the hypothesis presented provides a model that is amenable to experimental scrutiny. The next years will show what modifications we will have to make to accommodate new and critical findings.

\section{Acknowledgement}

The author's research on drug-induced hearing loss has been supported by grant NS-13792 from the National Institutes of Health.

\section{References}

Anniko, M. and Schacht, J. (1981): Phosphoinositides in the developing inner ear with references to brain, kidney and liver of the mouse. Int. J. Biochem. 13, 951-953.

Anniko, M., Takada, A. and Schacht, J. (1982): Comparative ototoxicities of gentamicin and netilmicin in three model systems. Am. J. Otolaryngol. 3, 422. 433.

Bennett, W.M., Plamp, C.E., Elliott, W.C., Parker, R.A. and Porter, G.A. (1982): Effects of basic amino acids and aminoglycosides on $\mathrm{H}-3$ gentamicin uptake in cortical slices of rat and human kidney. J. Lab. Clin. Med. 99, 156-162.

Berridge, M.J. (1984): Inositol trisphosphate and diacylglycerol as second messengers. Biochem. J. 220, 345-360.

Brown, J.E., Rubin, L.J., Ghalayini, A.J., Tarver, A.P., Irvine, R.F., Berridge, M.J. and Anderson, R.E. (1984): Myo-inositol polyphosphate may be a messenger for visual excitation in Limulus photoreceptors. Nature (London) 311 , $160-163$.

Burn, P., Rotman, A., Meyer, R.K. and Burger, M.M. (1985): Diacylglycerol in large actinin/actin complexes and in the cyloskeleton of activated platelets. Nature (London) 314 , 469-472.

Desrochers, C.S. and Schacht, J. (1982): Neomycin concentrations in inner ear tissues and other organs of the guinea pig after chronic drug administration. Acta Oto-Laryngol. 93, 233-236.

Escoubet, B., Amsallem, P., Ferrary, E. and Tran Ba Huy, P. (1985): Prostaglandin synthesis by the cochlea of the guinea pig - influence of aspirin, gentamicin and acoustic stimulation. Prostaglandins 29, 589-599.

Federspil, P. (1971): Übersicht über die in Deutschland beobachteten Fälle von Gentamicin-Ototoxizität. Z. Hals- Nasen- Ohrenhcilkd. 19, 328-331.

Federspil, P. (1979): Antibiotikaschäden des Ohres, pp. 98-105. Barth, Leipzig.

Hancock, R.E.W. (1981): Aminoglycoside uptake and mode of action with special reference to streptomycin and gentamicin. I. Antagonists and mutants. Antimicrob. Chemother. 8, $249-276$.

Hawkins, J.E. (1976): Drug ototoxicity. In: Handbook of Sensory Physiology, pp. 707-748. Editors: W.E. Keidel and W.D. Neff. Springer-Verlag, Berlin, Heidelberg.

Hayashi, R., Inoue, H., Amakawa, T. and Yoshioka, T. (1980): ${ }^{31}$ P NMR study of neomycin toxicity. Proc. Jpn Acad. 56B, 597-602.

Humes, H.D., Sastrasinh, M. and Weinberg, J.M. (1984): Calcium is a competitive inhibitor of gentamicin-renal membrane binding interactions and dietary calcium supplementation protects against gentamicin nephrotoxicity. J. Clin. Invest. 73, 134-147.

Jackson, G.G. and Arcieri, G. (1971): The ototoxicity of gentamicin in man: A survey and controlled analysis of clinical experience in the United States. J. Infect. Dis. 124, S130-137.

Josepovitz, C., Pastoriza-Muñoz, E., Timmerman, D., Scott, M., Feldman, S. and Koloyanides, G.J. (1982): Inhibition of gentamicin uptake in rat renal cortex in vivo by aminoglycosides and organic polycations. J. Pharmacol. Exp. Ther. 223, 314-321.

Juhn, S.K. and Youngs, J.N. (1976): The effect on perilymph of the alteration of serum glucose or calcium concentration. Laryngoscope 86, 273-279.

Kaloyanides, G.J. and Pastoriza-Muñoz, E. (1980): Aminoglycoside nephrotoxicity. Kidney Int. 18, 571-582.

Kilian, P. and Schacht, J. (1980): Sound stimulates labeling of polyphosphoinositides in the auditory organ of Noctuid moth. J. Neurochem. 34, 709-712.

Kroese, A.B.A. and Van den Bercken, J. (1982): Effects of ototoxic antibiotics on sensory hair cell functioning. Hearing Res. 6, 183-197.

Lassing, I. and Lindberg, U. (1985): Specific interaction between phosphatidylinositol 4,5-bisphosphate and profilactin. Nature (London) 314, 472-474.

Lodhi, S., Weiner, N.D. and Schacht, J. (1976): Interactions of neomycin and calcium in synaptosomal membranes and polyphosphoinositide monolayers. Biochim. Biophys. Acta 426, 781-785.

Lodhi, S., Weiner, N.D. and Schacht, J. (1979): Interactions of neomycin with monomolecular films of polyphosphoinositides and other lipids. Biochim. Biophys. Acta 426, 781-785.

Lodhi, S., Weiner, N.D., Mechigian, I. and Schacht, J. (1980): Ototoxicity of aminoglycosides correlated with their action on monomolecular films of polyphosphoinositides. Biochem. Pharmacol. 29, 597-601.

Marche, P., Koutouzov, S. and Girard, A. (1983): Impairment of membrane phosphoinositide metabolism by aminoglycoside antibiotics: Streptomycin, amikacin, kanamycin, dibe- 
kacin, gentamicin and neomycin. J. Pharmacol. Exp. Ther. 227. 415-420.

Matz, G.J. (1975): Toxic cochlear and vestibular disorders. In: The Nervous System, Vol. 3, pp. 333-349. Editor: D.B. Tower. Raven Press, New York.

Nishizuka, Y. (1984): Turnover of inositol phospholipids and signal transduction. Science 225, 1365-1370.

North. A. (1890): Two cases of poisoning by the oil of chenopodium. Am. J. Otol. 2, $197-200$.

Ohtsuki, K.. Ohtani. 1., Aikawa, T., Sato, Y., Anzai, T., Ouchi, J. and Saito, T. (1982): The ototoxicity and the accumulation in the inner ear of the various aminoglycoside antibio. tics. Ear. Res. Jpn. 13, 85-87.

Ohtani, T., Ohtsuki. K.. Aikawa, T., Sato, Y., Anzai, T., Ouchi. J. and Saito, J. (1985): Low ototoxicity and its mechanism of netilmicin. J. Oto-rhino-laryngol. Relat. Spec. 47, 84-89.

Orsulakova. A.. Stockhorst, E. and Schacht, J. (1976): Effect of neomycin on phosphoinositide labeling and calcium binding in guinea pig inner ear tissues in vivo and in vitro, $I$. Neurochem. 26, 285-290.

Rybak, L.P., Green T.P., Juhn S.K. and Morizono, T. (1984): Probenecid reduces cochlear effects and perilymph penetration of furosemide in chinchilla. J. Pharmacol. Exp. Ther. 230, 706-709.

Schacht, J. (1974): Interaction of neomycin with phosphoinositide metabolism in guinea pig inner ear and brain tissues. Ann. Otol. Rhinol. Laryngol. 83, 613-618.

Schacht, J. (1979): Isolation of an aminoglycoside receptor from guinea pig inner ear tissues and kidney. Arch. Otol. Rhinol. Laryngol. 224, 129-134.

Schacht, J. (1984): Phosphoinositides in the auditory system. In: Transmembrane Signaling and Sensation, pp. 89-97, Editor: F. Oosawa. Japan Societies Press, Tokyo.

Schacht, J., Lodhi, S, and Weiner, N.D. (1977): Effects of neomycin on polyphosphoinositides in inner ear tissues and monomolecular films. In: Membrane Toxicity, pp. 191-208. Editors: M.W. Miller and A.E. Shamoo. Plenum Press, New York.

Schibeci, A. and Schacht, J. (1977): Action of neomycin on the metabolism of polyphosphoinositides in the guinea pig kidney. Biochem. Phamiacol. 26, 1769-1774.

Schwabach, D. (1884): Über bleibende Störungen im Gehörorgan nach Chinin und Salicylgebrauch. Dtsch. Med. Wschr. $10,163-166$.

Shiozawa, K. and Yanagisawa, K. (1979): Effects of neomycin on the lateral-line organ of the mudpuppy. Proc. Jpn. Acad. Ser. B 55, 374-379.

Sokabe, M., Hayase, J. and Miyamoto, K. (1982): Neomycin effect on lysotriphosphoinositide channel as a model for an acute ototoxicity. Proc. Jpn. Acad. 58B, 177-180.

Stockhorst, E. and Schacht, J. (1977): Radioactive labeling of phospholipids and proteins by cochlear perfusion in the guinea pig and the effect of neomycin. Acta Oto-Laryngol. $83,401-409$.

Stupp. H.F. (1970): Untersuchung der Antibiotikaspiegel in den Innenohrflüssigkeiten und ihre Bedeutung für die spezifische Ototoxizität der Aminoglykosidantibiotika. Acta Oto-Laryngol. Suppl. 262. 1-85.

Tachibana, M., Anniko, M. and Schacht, J. (1983): Effects of perilymphatically perfused gentamicin on microphonic potential, lipid labeling, and morphology of cochlcar tissues. Acta Oto-Laryngol. 96, 31-38.

Takada, A. and Schacht, J. (1982): Calcium antagonism and reversibility of gentamicin-induced loss of cochlear microphonics in the guinea pig. Hearing Res. 8, 179-186.

Takada. A., Bledsoe, S.C. Jr. and Schacht, J. (1985): An energy-dependent step in aminoglycoside ototoxicity: Prevention of gentamicin ototoxicity during reduced endolymphatic potential. Hearing Res. 19. 245-251.

Tjernström, O., Banck, G., Belfrage, S., Juhlin, I., Nordström, L. and Toremalm, N.G. (1973): The ototoxicity of gentamicin. Acta Pathol. Microbiol. Scand. Scet. B S241, $73-78$.

Tran Ba Huy, P., Manuel, C., Meulemans, A., Sterkers, O. and Amiel, C. (1981): Pharmacokinetics of gentamicin in perilymph and endolymph of the rat as determined by radioimmunoassay. J. Infect. Dis. 143, 476-486.

Tran Ba Huy, P.. Meulemans. A., Wasef, M., Manuel, C.. Sterkers, O. and Amiel, C. (1983): Gentamicin persistence in rat endolymph and perilymph after a two day constant infusion. Antimicrob. Agents Chemother. 23, 344-346.

Tran Ba Huy, P., Bernard, P. and Schacht, J. (1986): Kinetics of gentamicin uptake and release in the rat: Comparison of inner ear tissues and fluids with other organs. J. Clin. Invest. (in press).

Voldrich, L. (1965): The kinetics of streptomycin, kanamycin and neomycin in the inner ear. Acta Oto-Laryngol. 60. $243-248$.

Vrabec, D.P., Cody, D.T. and Ulrich, J.A. (1965): A study of the relative concentrations of antibiotics in the blood, spinal fluid and perilymph in animals. Ann. Otol. (St. Louis) 74 , $688-705$.

Waksman, S.A. (1953): Streptomycin: Background, isolation, properties and utilization. Science 118, 259-266.

Wang, B.M., Weiner, N.D., Ganesan, M.G. and Schacht, J. (1984a): Interaction of calcium and neomycin with anionic phospholipid-lecithin liposomes: A differential scanning calorimetry study. Biochem. Pharmacol. 33, 3787-3791.

Wang, M.B.. Weiner, N.D.. Takada, A. and Schacht, J. (1984b): Characterization of aminoglycoside-lipid interactions and development of a refined model for ototoxicity testing. Biochem. Pharmacol. 33, 3257-3262.

Wersäll, J. (1986): Ototoxicity: An overview. Hearing Res. 22 , 293.

Wersäll, I. and Flock, A. (1964): Suppression and restoration of the microphonic output from the lateral line organ after local application of streptomycin. Life Sci. 3, 1151-1155.

Yanagisawa, K., Yoshioka, T., Inouc, H., Hayashi, F. and Tanaka, Y. (1982): Potassium effects on phosphatidylinositol phosphorylation in cochlear tissues. Neurosci. Lett. 9 , S113. 